\title{
Schon mal an Bettwanzen gedacht?
}

\begin{abstract}
Im letzten Jahrzehnt haben Parasiteninfektionen stark zugenommen. Neben Skabies und Flohstichen stellt der Befall durch Bettwanzen eine wichtige Differenzialdiagnose bei Juckreiz und urtikariellen Veränderungen der Haut dar.
\end{abstract}

Dr. Anthony Arayesh berichtet in „Der Hautarzt" über den Fall einer 27-jährigen Patientin mit Zweitwohnsitz in London. Die junge Frau stellte sich mit einem seit einigen Wochen bestehendem starkem Pruritus, fleckigen urtikariellen Erythemen und stecknadelkopfgroßen Papeln am gesamten Integument vor. Auf den initialen Verdacht einer Arzneimittelreaktion durch Paracetamol oder Aspirin ${ }^{\circledR}$, wur- de bereits mit einer systemischen Therapie mit Glukokortikosteroiden reagiert, die zunächst zu einer Abheilung der Hautveränderungen und der Beschwerden führte; jedoch kam es nach erneutem Aufenthalt in der Zweitwohnung zu einem Rezidiv der Symptome, so der Dermatologe.

\section{Am besten Nachweis des Insekts}

Differenzialdiagnostisch muss neben einer Arthropodenreaktion eine Nahrungsmittelunverträglichkeit, akute Urtikaria oder ein Arzneimittelexanthem bedacht werden. Zur Klärung der Ursache wurde ein Biopsat vom Rücken entnommen. Die histopathologische Untersuchung zeigt bei einer Cimicosis lediglich unspezifische Befunde, sodass schließlich der klinische

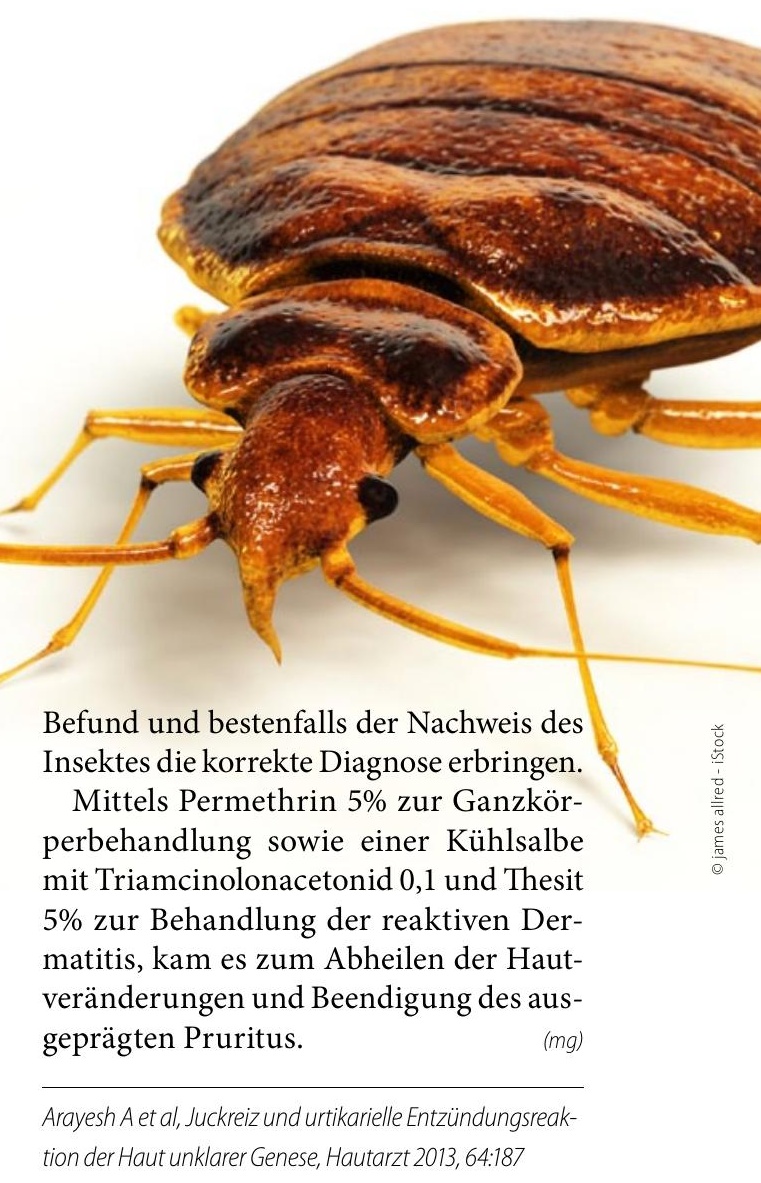

Impfung für alle Personen mit engem Kontakt zu Säuglingen wichtig“, betont Hofmann. Ab Vollendung des 60. Lebensjahres beinhalten die Standardimpfungen auch die jährliche Impfung gegen Influenza und die in der Regel einmalige Gabe eines Polysaccharid-Impfstoffs gegen Pneumokokken.

\section{Masern-Impfung nachholen!}

Letztes Jahr gab es in Deutschland nur 168 Masernerkrankungen, in den beiden Vorjahren waren es jedoch rund zehnmal so viele Fälle. Die STIKO empfiehlt die Masernimpfung deswegen inzwischen auch für Erwachsene. Alle, die nach 1970 geboren sind und einen unklaren Impfstatus haben bzw. in der Kindheit nicht oder nur einmal geimpft wurden, sollten eine Impfdosis, vorzugsweise einer MMR-Vakzine, erhalten.

Bei Frauen im gebärfähigen Alter muss ggf. der Schutz gegen Röteln komplettiert werden, gefordert werden zwei dokumentierte Impfungen. Wenn junge Frauen nicht im Alter zwischen 12 und 17 Jahren gegen HPV geimpft wurden, sollte Hofmann zufolge auch eine mögliche HPV-Nachholimpfung angesprochen werden.
Arbeitsmedizinische Impfindikationen sind keineswegs nur im Gesundheitsdienst, sondern auch in vielen anderen Branchen zu berücksichtigen. Eine Übersicht bietet die nebenstehende Tabelle. In der Regel sind diese Impfungen vom Arbeitgeber zu bezahlen. Auch Indikationsimpfungen müssen regelmäßig aufgefrischt werden, wenn die Indikation weiterhin besteht. Das gilt laut Hofmann für die Impfungen gegen FSME, Hepatitis A und B, Influenza, Meningokokken, Pertussis, Pneumokokken, Poliomyelitis, Tollwut und Typhus.

Stehen mehrere Impfungen an, kann das unter Umständen eine genaue Planung erfordern. Völlig unproblematisch sind Totimpfstoffe wie Polio, HAV und HBV sowie Toxoidimpfstoffe wie Tetanus, Diphtherie und Pertussis: Sie können in beliebiger Reihenfolge und auch gleichzeitig verabreicht werden.

Lebendimpfstoffe wie Masern, Mumps, Röteln sind dagegen entweder gleichzeitig oder aber im Abstand von vier Wochen zu geben, wie Hofmann betont. Außerdem müssen auf jeden Fall die Mindestabstände eingehalten werden, wenn von einer Vakzine mehrere Dosen notwendig sind.
„Liegt eine akute behandlungsbedürftige Erkrankung vor, besteht häufig eine Kontraindikation gegen eine Impfung“, schreibt Hofmann. Banale Infekte mit Temperaturen unter $38,6^{\circ} \mathrm{C}$ sind jedoch kein Hinderungsgrund.

Dasselbe gilt auch für Ekzeme und lokalisierte Hautinfektionen, die Einnahme von Antibiotika oder niedrig dosierten Glukokortikoiden sowie Krampfanfälle in der Familie oder frühere Fieberkrämpfe beim Impfling. Eindeutig kontraindiziert sind dagegen Lebendimpfstoffe bei Schwangeren.

\section{Vorbeugende Maßnahmen treffen}

„Keine absolute Kontraindikation“, so Hofmann, seien unerwünschte Arzneimittelwirkungen, die in engem zeitlichem Zusammenhang mit der vorausgegangenen Anwendung einer bestimmten Vakzine aufgetreten sind. „In diesem Fall sollten jedoch vorbeugende Maßnahmen getroffen werden, z. B. eine antihistaminische Vorbehandlung eine Stunde vor der Impfung und ggf. eine Glukokortikoidgabe."

(Beate Schumacher)

Hofmann F, MMW-Fortschr. Med. 2012,

Sonderheft 3: 52-58 